\title{
Genetic characterization of ESBL-producing strains of Klebsiella pneumoniae from Tehran hospitals
}

\author{
Mohammad Mehdi Feizabadi ${ }^{1}$, Samira Mohammadi-Yeganeh ${ }^{1}$, Akbar Mirsalehian ${ }^{1}$, Poorya Azimi ${ }^{1}$, \\ Seyed Mohammad Mirafshar ${ }^{1}$, Mohaddeseh Mahboobi ${ }^{2}$, Firoozeh Nili ${ }^{3}$, Davood Yadegarinia ${ }^{4}$ \\ ${ }^{1}$ Department of Microbiology, School of Medicine, Medical Science, University of Tehran, Tehran, Iran \\ ${ }^{2}$ Department of Microbiology, Barij-Essence Pharmaceutical Industry, Kashan, Iran \\ ${ }^{3}$ Department of Pediatrics Disease, School of Medicine, Medical Science, University of Tehran, Tehran, Iran \\ ${ }^{4}$ Infectious Disease and Tropical Disease Research Center, Shahid Beheshti University of Medical Science, \\ Tehran, Iran
}

\begin{abstract}
Introduction: This study was conducted to determine the genetic characterization of extended-spectrum beta-lactamase (ESBL) producing strains of Klebsiella pneumoniae isolated from Iranian patients in hospitals in Tehran.

Methodology: Antibiotic susceptibility of 104 isolates was determined using the disk diffusion test. The Minimum Inhibitory Concentrations (MICs) of imipenem and meropenem were determined for isolates showing reduced susceptibility to carbapenems. The phenotypic confirmatory test (PCT) was used to screen the isolates for ESBL production. PCR was used to detect $b l a_{\mathrm{SHV}}, b l a_{\mathrm{TEM}}$ and $b l a_{\mathrm{CTX}-\mathrm{M}}$ and the amplicons from selected clones were sequenced. Isolates producing ESBLs were analyzed by pulsed-field gel electrophoresis (PFGE).

Results: One isolate showed resistance to imipenem (MIC $=16 \mu \mathrm{g} / \mathrm{ml}$ ). Resistance to amikacin and ciprofloxacin was $44.2 \%$ and $25.0 \%$, respectively. ESBL production was detected in $72.1 \%(\mathrm{n}=75)$ of isolates. The prevalence of $b l a_{\mathrm{SHV}}$, $b l a_{\mathrm{TEM}}$ and $b l a_{\mathrm{CTX}-\mathrm{M}}$ genes among the isolates was $55.7 \%(\mathrm{n}=58), 30.7 \%(\mathrm{n}=32)$ and $45.2 \%(\mathrm{n}=47)$, respectively. The sequencing revealed the amplicons corresponding to bla (TEM-1, TEM-79, SHV-1, SHV-12, SHV-31, CTX-M-15) genes. While the $b l a_{\mathrm{CTX}-\mathrm{M}-15}$ is the dominant gene among the Iranian isolates, we detected the $b l a_{\mathrm{SHV}}$ 31 and $b l a_{\text {TEM-79 }}$ genes for the first time in the country. PFGE differentiated the 71 ESBL-producing isolates into 62 different genotypes. Clonal dissemination of ESBLs was found in the neonatal intensive care unit and intensive care unit of one hospital.

Conclusion: The findings are evidence of the spread of multi-resistant clones of ESBL producers in Tehran hospitals.
\end{abstract}

Key words: Klebsiella pneumoniae; ESBL, PCR, PFGE, FIGE, Iran

J Infect Dev Ctries 2010; 4(10):609-615.

(Received: 31 March 2010 - Accepted: 17 May 2010)

Copyright (C) 2010 Feizabadi et al. This is an open-access article distributed under the Creative Commons Attribution License, which permits unrestricted use, distribution, and reproduction in any medium, provided the original work is properly cited.

\section{Introduction}

With the widespread use of broad-spectrum antibacterial agents, problems in treatment of infections with drug resistant organisms, in particular those acquiring resistance to beta-lactams, including broad-spectrum cephalosporins, has increased. Resistance to the new cephalosporins is mediated by production of extended-spectrum beta-lactamases (ESBLs). The ESBL-producing isolates of Klebsiella pneumoniae have been associated with numerous outbreaks of nosocomial infections throughout Europe, the United States of America [1] and the Far East [2], though such outbreaks have recently been reported in Asia [3,4] including Iran [5-7]. Strains of $K$. pneumoniae producing ESBLs are widespread in Tehran hospitals but relatively little is known about the epidemiology and genetic background of the involved organism. Dissemination of ESBLproducing $K$. pneumoniae in a hospital may be a complex event involving several modes of epidemic spread. Molecular typing techniques, particularly macrorestriction patterns of total genomic DNA, obtained by pulsed-field gel electrophoresis (PFGE), are now widely accepted as powerful epidemiological tools and have been applied for the molecular characterization in outbreaks $[8,9]$.

The main aims of this study were to assess the susceptibility of $K$. pneumoniae strains isolated from the Iranian patients to different antimicrobial agents and to characterize the genes encoding the ESBL phenotypes. Since little data was available on the genetic relationships among the Iranian isolates of $K$. 
pneumoniae, we also applied PFGE to investigate the possibility of clonal outbreaks or heterogeneity of ESBL-producing strains isolated from different wards of the study hospitals.

\section{Methodology}

Bacterial isolates and phenotypic characterization

Collectively, a total of 104 consecutive nonduplicate isolates of $K$. pneumoniae were collected from September 2006 to June 2007 from four university hospitals in Tehran. Two general hospitals located in the city center (Hospital A with 1,230 beds and Hospital D with 338 beds), 2 children's hospitals located in the city center (Hospital B with 350 beds), and one hospital in the north of the capital (Hospital C with 210 beds) were included in the study.

Bacterial isolates were identified using the standard procedures [10]. They were tested against the following antibiotics using the Clinical and Laboratory Standards Institute (CLSI) protocol [11]: imipenem, meropenem, amikacin, ciprofloxacin, levofloxacin, piperacillin-tazobactam, amoxicillinclavulanic acid, ampicillin-sulbactam, aztreonam, cefotaxime, ceftazidime, ceftriaxone and cefepime. $K$. pneumoniae ATCC 700603 was used as the control in all experiments. The Minimum Inhibitory Concentrations (MICs) of imipenem (MAST,Merseyside, UK) against isolates showing reduced susceptibility to this carbapenem was determined using micro-broth dilution assay.

Isolates showing resistance to at least one of the thirrd generation cephalosporins (3GCs) or aztreonam were tested for ESBL production using the phenotypic confirmatory test (PCT) as instructed by CLSI [11]. K. pneumoniae ATCC 700603 was used as the positive control and Escherichia coli ATCC 25922 was used as the negative control.

\section{PCR amplification of beta-lactamase genes}

DNA of all isolates was extracted by the boiling method. The bla $a_{S H}$ and $b l a_{T E M}$ were amplified as described previously [5]. Plasmid DNA was extracted by miniprep kit (Metabion, Martinsried, Germany) and targeted for the $b l a_{\text {CTX-M }}$ gene using the primers described by Lee et al. [12] under the following conditions: $94^{\circ} \mathrm{C}$ for 3 minutes, and 35 cycles of $94^{\circ} \mathrm{C}$ for 30 seconds, $63^{\circ} \mathrm{C}$ for 1 minute, $72^{\circ} \mathrm{C}$ for 1 minute and a final extension in $72^{\circ} \mathrm{C}$ for 10 minutes. K. pneumoniae ATCC 700603, E. coli ATCC 35218 and $K$. pneumoniae 7881 were used as positive controls for bla $a_{S H V}, b l a_{T E M}$ and bla $a_{C T X-M}$, respectively.
The latter isolates were kindly provided by Professor Patrice Nordmann of Institut National de la Santé et de la Recherche Médicale, Paris, France.

\section{Pulsed Field Gel Electrophoresis (PFGE)}

The Genomic DNA was extracted using the protocol described by Bouallegue-Godet et al. [8] with some modifications. The embedded DNA in plugs was digested with XbaI (Fermentas, Vilinus, Lithuania) for 4 hours at $37^{\circ} \mathrm{C}$. The digested DNAs were separated in $1 \%$ agarose gel by field inversion gel electrophoresis (FIGE), a prototype of PFGE (Faculty of Engineering, University of Tehran). Conditions were as follows: pulse time ramped linearly from 1 to $35 \mathrm{~s}$, run time 20 hours, temperature $14^{\circ} \mathrm{C}$, and constant voltage of $6 \mathrm{~V} / \mathrm{cm}$. The gels were stained with ethidium bromide and DNA patterns were photographed with UVP gel documentation (UVP,Cambridge, UK) (Figure 1). The DNA patterns were analysed with Zhen Negar software (Faculty of Mathematic and Computing Sciences, Sharif University,Tehran, Iran) and the strains were clustered by the unweighted pair group method with arithmetic averages strategy [13]. The DNA banding patterns were interpreted as instructed by Tenover et al. [14].

\section{Sequencing of PCR products}

Based on the results of PFGE analysis, the amplicons from isolates with identical DNA banding patterns were selected and sequenced. The sequences were analyzed using the BLAST program (www.ncbi.nlm.nih.gov=BLAST) and submitted to GenBank.

\section{Results}

Resistance of K. pneumoniae isolates

In total, 104 isolates of $K$. pneumoniae were isolated from urine $(\mathrm{n}=33)$, respiratory tract $(\mathrm{n}=$ $14)$, blood $(n=18)$, wound exudates $(n=22)$ and other clinical specimens $(n=17)$. The majority of the strains $(79.8 \%)$ were cultured from hospitalized patients and the remainder (20.2\%) belonged to outpatients. Using the PCT method, ESBL production was found in $75(72.1 \%)$ isolates.

All ESBL-positive isolates were susceptible to meropenem, but one isolate showed resistance to imipenem (MIC $=16 \mu \mathrm{g} / \mathrm{mL}$ ). Following carbapenems, the lowest rates of resistance in ESBLproducing isolates were observed for amikacin (21.4\%), piperacillin/tazobactam (21.3\%), levofloxacin $(25.3 \%)$ and ciprofloxacin $(28.0 \%)$. The 
highest rate of resistance belonged to aztreonam (92.0\%), cefotaxime, ceftazidime $(84.0 \%)$ and cefepime $(76.0 \%)$. Piperacillin/tazobactam was more potent than ampicillin-sulbactam and amoxicillin/clavulanic acid; results showed that
$69.3 \%$ of ESBL isolates were susceptible to this combination. The resistance rate for cefepime was 76\%. In ESBL-positive isolates, 21 isolates (28\%) had concomitant resistance to ciprofloxacin.

Table 1. Clones of $K$. pneumoniae detected by PFGE in regard to their ESBL phenotypes and the relevant wards

\begin{tabular}{|c|c|c|c|c|}
\hline Clone & Isolates Number & Sample & Hospital wards & ESBL phenotype \\
\hline \multirow[t]{7}{*}{ A } & 305 & Eye exudates & NICU & SHV-1, CTX-M-15 \\
\hline & 308 & Eye exudates & NICU & SHV-1, CTX-M-15 \\
\hline & 309 & Eye exudates & NICU & SHV-1, CTX-M-15 \\
\hline & 359 & Bronchial & $\mathrm{NICU}$ & SHV-1, CTX-M-15 \\
\hline & 302 & Wound & Surgery & SHV-1, CTX-M-15, TEM-1 \\
\hline & 312 & Bronchial & NICU & SHV-1, CTX-M-15 \\
\hline & 313 & Bronchial & NICU & SHV-1, CTX-M-15 \\
\hline \multirow[t]{2}{*}{ B } & 344 & Blood & ICU & CTX-M-15 \\
\hline & 358 & Abdominal exudates & ICU & SHV-1, CTX-M-15 \\
\hline \multirow[t]{2}{*}{$\mathrm{C}$} & 329 & Blood & ICU & TEM-79, CTX-M-15 \\
\hline & 306 & Blood & ICU & СTX-M-15, TEM-79,SHV-12 \\
\hline \multirow[t]{2}{*}{ D } & 350 & Eye exudates & NICU & SHV-31 \\
\hline & 375 & Umbilical exudates & NICU & SHV-31 \\
\hline
\end{tabular}

Prevalence of bla $_{S H V}$, bla $_{T E M}$, and bla ${ }_{C T X-M}$ genes and sequencing results

PCR showed that $55.7 \%(\mathrm{n}=58), 30.7 \%(\mathrm{n}=32)$ and $45.2 \%(\mathrm{n}=47)$ of isolates contained bla $a_{\mathrm{SHV}}$, $b l a_{\mathrm{TEM}}$, and $b l a_{\mathrm{CTX}-\mathrm{M}}$ genes, respectively. Coexistence of these genes was detected in $26(34.7 \%)$ of isolates. Of 104 isolates tested for the above genes, only three isolates were not positive in PCT but produced amplicons corresponding to $b l a_{\mathrm{SHV}}$ and $b l a_{\mathrm{TEM}}$. They were negative for $b l a_{\text {СTX-м. }}$.

With the exception of two isolates, the results of sequencing showed that all bla $a_{\mathrm{TEM}}$ amplicons belonged to $b l a_{\text {TEM-1 }}$ (GenBank accession numbers GU734690-GU73469097). The excepted isolates cultured from blood specimens and possessed $b l a_{\mathrm{TEM}}$ 79 (Table 1). There was more diversity among sequences obtained for bla 
Figure 1. Phlogenetic relationships among isolates of $K$. pneumoniae using data obtained by PFGE. The strains were clustered by the unweighted pair group method with arithmetic averages (UPGMA). The scale indicates the percentage of genetic distances. Hospitals: A; Emam Khomeini hospital, B; Markaz Tebbi Kudakan Hospital, C; Mofid Hospital, D; Sina hospital
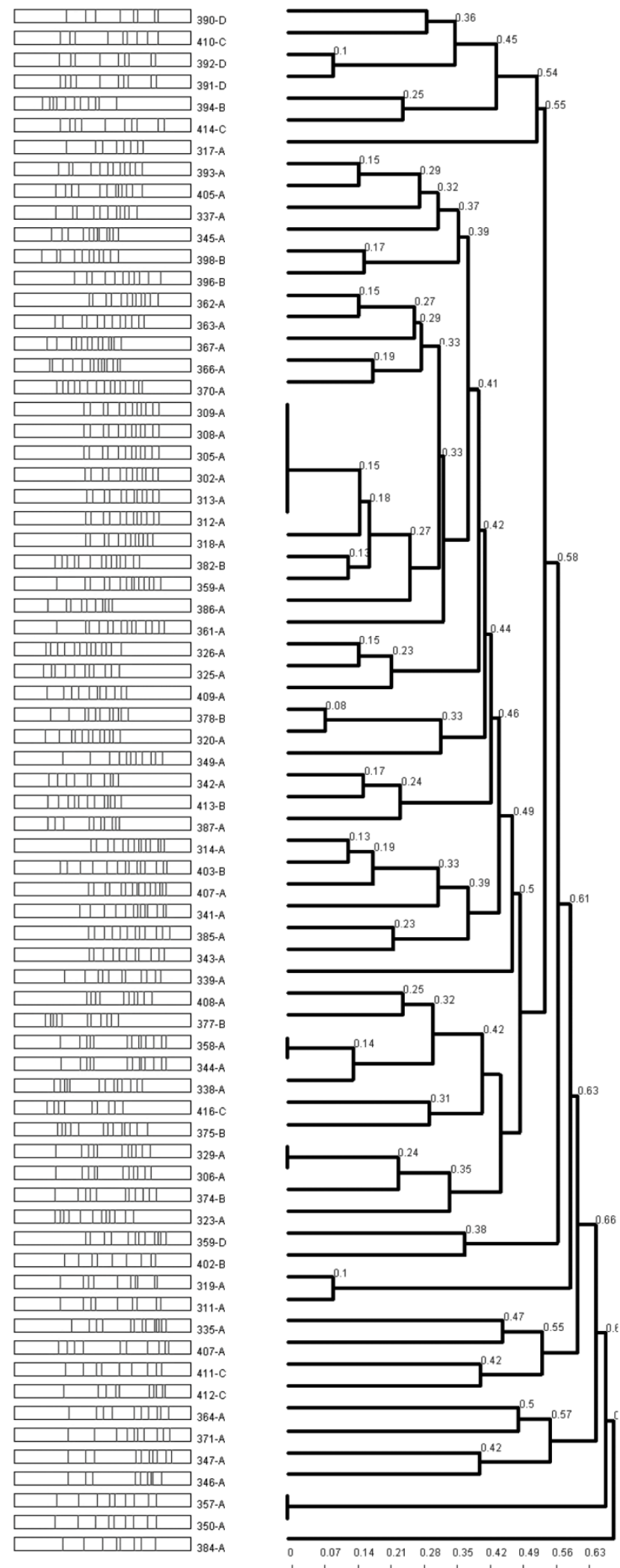
genes and these belonged to $\operatorname{bla}_{(\mathrm{SHV}-1, \mathrm{SHV}-12, \mathrm{SHV}-31)}$ (Table 1). The amplicons obtained for $b l a_{\text {СтX-м }}$ were characterized as $b l a_{\text {CTX-M-15 }}$ after sequencing and the relevant sequences were submitted to GenBank under accession numbers HM002646-HM002660.

\section{PFGE analysis}

Analysis of ESBL-producing strains by PFGE showed that the majority of isolates were not related (Figure 1). Four isolates did not yield sufficient DNA. These isolates belonged to outpatients and were excluded from PFGE analysis.

Of 71 ESBL-producing strains analyzed by PFGE, isolates 51, 11, 5 and 4 belonged to hospitals $\mathrm{A}, \mathrm{B}, \mathrm{C}$ and $\mathrm{D}$, respectively. The numbers of distinctive patterns identified in these hospitals were 39, 11, 5 and 4. Therefore, 13 strains from hospital A were clustered in 4 groups (Table 1). These isolates were cultured from patients in a neonatal intensive care unit (NICU) $(\mathrm{n}=8)$, an intensive care unit (ICU) $(\mathrm{n}=4)$, and a surgery unit $(\mathrm{n}=1)$. One isolate collected from the surgery unit and 6 isolates from NICU were identical. However, genetically distinct strains were also detected in hospital A, which is the largest health care setting in Iran. The ICU isolates were collected from blood and abdominal exudates.

The remaining isolates $(n=58)$ were distributed through the dendrogram at different genetic distances (Figure 1).

\section{Discussion}

In Asia, the prevalence of ESBL-positive $K$. pneumoniae isolates varies within different regions. The ESBL frequency in neighboring countries varied from $85 \%$ in Russia, $66.7 \%$ in India [4] , 54.7\% and $61 \%$ in Turkey [3,15], $41 \%$ in United Arab Emirates [16] and $31.7 \%$ in Kuwait [17,] but it is lower in America and Europe $[15,18]$. In comparison with our previous studies [5,7], in this study, we found ESBL production in $72.1 \%$ of isolates $(n=75)$ because most of these isolates were collected from hospitalized patients and ESBL production is more common among hospitalized patients $(n=66,79.5 \%)$ than outpatients $(n=9,42.9 \%)[18]$. In agreement with recent trends [6], ESBL phenotypes showed remarkable increase in the CTX-M phenotype. Moreover, we found a new type of SHV genotype $\left(b l a_{\mathrm{SHV}-31}\right)$ which had caused a large outbreak in the Netherlands [19]. This illustrates the necessity for continued phenotypic surveillance for ESBLproducing strains of $K$. pneumoniae. Three isolates were not positive in PCT but produced amplicons corresponding to $b l a_{\mathrm{TEM}}$ and $b l a_{\mathrm{SHV}}$ genes, which shows that the sensitivity of the PCT assay is not $100 \%$ and that the PCR assay is more reliable [2].

The high rate of ESBL enzymes in Tehran hospitals probably is due to the frequent use of $3 \mathrm{GCs}$ or the spread of resistant plasmids among the bacterial isolates in the hospital environments as explained previously [20].

Cefepime is more stable to hydrolysis by ESBLs than the 3GCs [21]; however, the rate of resistance to this fourth-generation cephalosporin $(76 \%)$ was considerably high in this study. This antibiotic has been administered in recent years and widely used against different infections in Iran. It seems that cefepime should not be used as the first choice of therapy against ESBL-producing organisms.

Similar to other studies, we found that ESBLproducing $K$. pneumoniae strains are associated with high aminoglycoside resistance [22]. Amikacin remained the most effective aminoglyoside against $K$. pneumoniae.

The spread of $K$. pneumoniae with resistance to both quinolones and broad-spectrum beta-lactams would severely limit treatment options. Levofloxacin, which is not being prescribed in Iran, was more effective than ciprofloxacin since it was active against 24 isolates with a ciprofloxacin resistance phenotype.

The high rate of resistance to amoxicillin/clavulanic acid among ESBL-producing isolates $(\mathrm{n}=50,66.7 \%)$ is probably due to the frequent use of this antibiotic to treat paediatric otitis media and gastritis caused by Helicobacter pylori [23]. Such rate of resistance was also reported from Malaysian hospitals [24].

The carbapenems, in combination with aminoglycosides, are the final therapeutic option against ESBL-producing isolates [25]. Unfortunately, we found the first imipenem resistant isolate recovered from the wound of a surgery patient. To our knowledge, such resistance has not been reported in Iran before. Similar cases of imipenem resistant $K$. pneumoniae were reported from Turkey in 2006 and 2007 [3,26]. In most European countries, Latin America, and East Asia, CTX-M variants have displaced TEM and SHV enzymes as the predominant beta-lactamases produced by Gramnegative bacteria such as $K$. pneumoniae $[4,27]$ and our data confirms this document.

The high genetic diversity detected in the collection of isolates from hospital A can be attributed to the diversity of sources that is being 
introduced to this hospital. It is the largest hospital in Iran and patients are referred to it from nearly all parts of the country. However, the PFGE profiles demonstrated a few clonal dissemination of ESBLpositive strains in hospital A, which shows that vertical spread of resistant isolates had occurred in this hospital. The isolates with identical patterns suggest that clonal spread also played a role in the dissemination of ESBL-producing isolates in the NICU and ICU. It appears that a multi-drug resistant $K$. pneumoniae clone detected in the NICU was related to a clone isolated from women's surgery, as both of them produced similar PFGE patterns.

In conclusion, carbapenems still show the highest potency against $K$. pneumoniae in Iran. However, emerging of resistance to this group of antibiotics, in particular resistance to imipenem, may limit their prescription in the future. The tremendous increase in prevalence of ESBLs (at least 26.7\%) is the second concern since the majority of them were multiresistant to other antibiotics. Importantly, inactivation of beta-lactamase inhibitors, in particular clavulanic acid, has reduced its efficacy in combination treatment with beta-lactams against ESBL-producing isolates.

\section{Acknowledgment}

This work was supported by a grant from Tehran University of Medical Sciences (Grant 3065). The expenses of sequencing were covered by a grant from the Infectious Diseases and Tropical Medicine Research Center at Shahid Beheshti University of Medical Sciences. The authors thank Haudi Dehdashti for his editing work on the manuscript.

\section{References}

1. Bush K (2008) Extended-spectrum beta-lactamases in North America. 1987-2006. Clin Microbiol Infect 14:134-143.

2. Coque TM, Baquero F, Canton R (2008) Increasing prevalence of ESBL-producing Enterobacteriaceae in Europe. Euro Surveill 13(47).

3. Gur D, Hascelik G, Aydin N, Telli M, Gültekin M, Ogülnç D, Arikan OA, Uysal S, Yaman A, Kibar F, Gülay Z, Sumerkan B, Esel D, Kayacan CB, Aktas Z, Soyletir G, Altinkanat G, Durupinar B, Darka O, Akgün Y, Yayla B, Gedikoglu S, Sinirtas M, Berktas M, Yaman G. (2009) Antimicrobial resistance in gram-negative hospital isolates: results of the Turkish HITIT-2 Surveillance Study of 2007. J Chemother 21: 383-389.

4. Hawkey PM (2008) Prevalence and clonality of extendedspectrum beta-lactamases in Asia. Clin Microbiol Infect 14: 159-165.

5. Shahcheraghi F, Moezi H, Feizabadi MM (2007) Distribution of TEM and SHV Beta-lactamase genes among Klebsiella pneumoniae strains isolated from patients in Tehran. Med Sci Monit 13:247-250.

6. Feizabadi MM, Delfani S, Raji N, Majnooni A, Aligholi M, Shahcheraghi F, Parvin M, Yadegarinia D (2009)
Distribution of bla(TEM), bla(SHV), bla(CTX-M) Genes Among Clinical Isolates of Klebsiella pneumoniae at Labbafinejad Hospital, Tehran, Iran. Microb Drug Resist 16: 49-53.

7. Feizabadi MM, Etemadi G, Yadegarinia D, Rahmati M, Shabanpoor S, Bokaei S (2006) Antibiotic-resistance patterns and frequency of extended-spectrum betalactamase-producing isolates of Klebsiella pneumoniae in Tehran. Med Sci Monit 12: 362-365.

8. Bouallegue-Godet O, Grimont F, Ben Salem Y, Saidani M, Mzoughi R, Sboui H, Grimont PA (2005) Investigation of the clonal dissemination of Klebsiella pneumoniae isolates producing extended-spectrum beta-lactamases in a neonatal ward, Sousse, Tunisia. Pathol Biol 53:75-80.

9. Mesko Meglic K, Koren S, Palepou MF, Karisik E, Livermore DM, Pike R, Andlovic A, Jeverica S, KrizanHergouth V, Müller-Premru M, Seme K; Slovenian ESBL Study Group, Woodford N.(2009) Nationwide survey of CTX-M-type extended-spectrum beta-lactamases among Klebsiella pneumoniae isolates in Slovenian hospitals. Antimicrob Agents Chemother 53:287-291.

10. JF MacFaddin (1999) Biochemical tests for identification of medical bacteria. Philadelphia: Lippincott Williams and Wilkinson.

11. CLSI Performance Standards for Antimicrobial Susceptibility Testing (2006) Vol. Informational Supplement, 16th edn M100-S16.

12. Lee S, Park YJ, Kim M, Lee HK, Han K, Kang CS, Kang MW (2005) Prevalence of Ambler class A and D betalactamases among clinical isolates of Pseudomonas aeruginosa in Korea. J Antimicrob Chemother 56: 122-127.

13. Wofford D, Olsen G, Waddel I (1996) Molecular Systemics. Massachusetts: Sinauer Associates Inc. Sunderland, USA.

14. Tenover FC, Arbeit RD, Goering RV, Mickelsen PA, Murray BE, Persing DH, Swaminathan B (1995) Interpreting chromosomal DNA restriction patterns produced by pulsed-field gel electrophoresis: criteria for bacterial strain typing. J Clin Microbiol 33: 2233-2239.

15. Perez F, Endimiani A, Hujer KM, Bonomo RA (2007) The continuing challenge of ESBLs. Curr Opin Pharmacol 7: 459-469.

16. Al-Zarouni M, Senok A, Rashid F, Al-Jesmi SM, Panigrahi D (2008) Prevalence and antimicrobial susceptibility pattern of extended-spectrum beta-lactamase-producing Enterobacteriaceae in the United Arab Emirates. Med Princ Pract 17: 32-36.

17. Mokaddas EM, Abdulla AA, Shati S, Rotimi VO (2008) The technical aspects and clinical significance of detecting extended-spectrum beta-lactamase-producing Enterobacteriaceae at a tertiary-care hospital in Kuwait. J Chemother 20: 445-451.

18. Khanfar HS, Bindayna KM, Senok AC, Botta GA (2009) Extended spectrum beta-lactamases (ESBL) in Escherichia coli and Klebsiella pneumoniae: trends in the hospital and community settings. J Infect Dev Ctries 3: 295-299.

19. Mazzariol A, Roelofsen E, Koncan R, Voss A, Cornaglia G (2007) Detection of a new SHV-type extended-spectrum beta-lactamase, SHV-31, in a Klebsiella pneumoniae strain causing a large nosocomial outbreak in The Netherlands. Antimicrob Agents Chemother 51: 1082-1884.

20. Miranda G, Castro N, Leanos B, Valenzuela A, GarzaRamos U, Rojas T, Solórzano F, Chihu L, Silva J.(2004) Clonal and horizontal dissemination of Klebsiella 
pneumoniae expressing SHV-5 extended-spectrum betalactamase in a Mexican pediatric hospital. J Clin Microbiol 42:30-35.

21. Labombardi VJ, Rojtman A, Tran K (2006) Use of cefepime for the treatment of infections caused by extended spectrum beta-lactamase-producing Klebsiella pneumoniae and Escherichia coli. Diagn Microbiol Infect Dis 56: 313-315.

22. Alcantar-Curiel D, Tinoco JC, Gayosso C, Carlos A, Daza C, Perez-Prado MC, Salcido L, Santos JI, Alpuche-Aranda CM.(2004) Nosocomial bacteremia and urinary tract infections caused by extended-spectrum beta-lactamaseproducing Klebsiella pneumoniae with plasmids carrying both SHV-5 and TLA-1 genes. Clin Infect Dis 38:10671074.

23. Avci IY, Kilic S, Acikel CH, Ucar M, Hasde M, Eyigun CP, Pahsa A, Cetiner S (2006) Outpatient prescription of oral antibiotics in a training hospital in Turkey: trends in the last decade. J Infect 52: 9-14.

24. Loh LC, Chin HK, Chong YY, Jeyaratnam A, Raman S, Vijayasingham P, Thayaparan T, Kumar S (2007) Klebsiella pneumoniae respiratory isolates from 2000 to 2004 in a
Malaysian hospital: characteristics and relation to hospital antibiotics consumption. Singapore Med J 48: 813-818.

25. Paterson DL (2000) Recommendation for treatment of severe infections caused by Enterobacteriaceae producing extended-spectrum beta-lactamases (ESBLs). Clin Microbiol Infect 6: 460-463.

26. Aktas Z, Bal C, Midilli K, Poirel L, Nordmann P (2006) First IMP-1-producing Klebsiella pneumoniae isolate in Turkey. Clin Microbiol Infect 12: 695-6.

27. Paterson DL, Bonomo RA (2005) Extended-spectrum $\beta$ lactamases: a Clinical Update. Clin Microbiol Rev 18: 657686.

\section{Corresponding author}

Mohammad Mehdi Feizabadi

Department of Microbiology, School of Medicine

Poorsina Street, Tehran, Iran

Telefax: 0098-21-88955810

Email: mfeizabadi@sina.tums.ac.ir

Conflict of interests: No conflict of interests is declared. 